\title{
Indices biométriques et paramètres de croissance du tilapia Oreochromis niloticus (Linnaeus, 1758) exposé aux pesticides agricoles dans les retenues d'eau du Nord-Bénin
}

\author{
Ibrahim IMOROU TOKO ${ }^{*}$, O.E. Rodrigue PELEBE ${ }^{1}$, Roland TONATO ${ }^{1}$, \\ N. Léa GUEDEGBA ${ }^{1,2}$, T. Prudencio AGBOHESSI ${ }^{1}$ et Patrick KESTEMONT ${ }^{2}$ \\ ${ }^{1}$ Laboratoire de Recherche en Aquaculture et Ecotoxicologie Aquatique (LaRAEAq), Faculté \\ d'Agronomie (FA), Université de Parakou (UP), Bénin. \\ ${ }^{2}$ Unité de Recherche en Biologie Environnementale et Evolutive (URBE), Faculté des Sciences, Université de \\ Namur, Belgique. \\ *Auteur correspondant, E-mail: iimorou_toko@hotmail.com; Tél: 0022996132150.
}

\section{REMERCIEMENTS}

La présente étude a été réalisée grâce au concours financier de l'ARES-CCD à travers le projet AquaTox-Bénin et du Programme d'Appui à l'Enseignement Supérieur (PAES) de l'UEMOA à travers le Projet $N^{\circ}$ P-Z1-IAD-O02.

\section{RESUME}

Les retenues d'eau constituent généralement les réceptacles finaux des pesticides agricoles utilisés dans le bassin cotonnier béninois. La présente étude vise à étudier l'état de santé des poissons dans ces milieux à partir des indices biométriques et des paramètres de croissance. Ainsi, des alevins de Oreochromis niloticus y ont été élevés en enclos et en happas durant 50 jours à Batran et à Songhai (témoin). Chez les mâles, les indices gonado-somatique, hépato-somatique et de condition n'ont pas significativement ( $p>0,05)$ varié d'une retenue à une autre, de même que l'IGS et l'IHS en happas chez les femelles. Cependant, l'IGS et l'IHS des femelles en enclos sont significativement plus élevés à Batran qu'à Songhai. Mais aussi bien en enclos qu'en happas, l'indice de condition des femelles est supérieur à Songhai qu'à Batran $(\mathrm{p}<0,05)$. Dans la retenue d'eau témoin les meilleurs performances de croissance sont obtenues et l'élevage en happas semble meilleur qu'en enclos dans cette retenue. Cependant, à Batran les poissons élevés en enclos ont mieux grandi que ceux des happas. A Songhaï comme à Batran, les indices biométriques sont généralement meilleurs dans les happas que dans les enclos qui exposent probablement plus les poissons aux pesticides piégés dans la vase.

(C) 2018 International Formulae Group. All rights reserved.

Mots clés: Pesticides, retenues d'eau, santé des poissons, biomarqueurs.

\section{Biometric index and growth parameters of tilapia Oreochromis niloticus} (Linnaeus, 1758) exposed to agricultural pesticides in water reservoirs located in northern Benin 


\section{ABSTRACT}

Water reservoirs are generally the final receptacles for agricultural pesticides used in the Beninese cotton basin. The present study aims to study the health status of fish in these environments from biometric index and growth parameters. Thus, juveniles of Oreochromis niloticus were raised in pens and happas for 50 days in Batran and Sanghai (Control) water reservoirs. In males, the gonado-somatic, hepatostatic and condition index did not significantly ( $p>0.05$ ) varied from one reservoir to another, as did GSI and HSI in happas for females. However, the GSI and HSI of females in pens are significantly higher for fish in Batran than in Songhai. In both pens and happas, the condition index of females is higher at Songhai than Batran (p $<0.05$ ). In the control water reservoir the best growth performances are obtained and breeding fish in happas seems better than in pens in this reservoir. However, in Batran the fish raised in pens have grown better than those in happas. In Songhai and Batran, the biometric indexes are generally betters in the happas than in pens that are likely to expose more fish to the pesticides trapped in the mud.

(C) 2018 International Formulae Group. All rights reserved.

Keywords: Pesticides, water reservoir, fish health, biomarkers.

\section{INTRODUCTION}

Les tilapias constituent le troisième groupe de poissons le plus élevé au monde après les Cyprinidés et les Salmonidés (Al Dilaimi, 2009). La production mondiale du tilapia a connu un véritable boom puisqu'en trente ans elle a été multipliée par 15. Au cours de la dernière décennie, elle a plus que doublée, passant de 830.000 tonnes en 1990 à 3,5 millions de tonnes en 2008 (FAO, 2010). La Chine a joué un rôle majeur dans cette croissance, étant à l'origine de plus de 60 pour cent de la production aquacole mondiale (FAO, 2016). La production africaine, destinée aux marchés nationaux, a également augmenté, avec près de 430.000 tonnes de tilapia d'élevage produits en 2008, soit deux fois plus qu'en 2000 (FAO, 2010). L'Egypte est le deuxième producteur mondial de cette espèce et le premier producteur africain (FAO, 2016).

$\mathrm{Au}$ Bénin, les tilapias, notamment Oreochromis niloticus occupent une place de choix dans les exploitations piscicoles du fait de la forte demande des consommateurs. Ils sont particulièrement abondants dans les retenues d'eau (barrages et surcreusements) où ils représentent plus de $60 \%$ des captures (Adam Sanni, 2011). Dans le Nord du Bénin, ces retenues d'eau qui représentent environ $83 \%$ des retenues d'eau du pays, fournissent plus de 60\% (environ 800 tonnes) du poisson frais consommé annuellement (Imorou Toko et al., 2011), bien qu'elles soient très souvent exposées aux biocides agricoles du fait de leur proximité aux exploitations agricoles, notamment cotonnières (Agbohessi, 2014 ; Adechian et al., 2015). En effet, l'obtention de meilleurs rendements dans les exploitations cotonnières qui constituent la principale culture de rente du pays est tributaire de l'utilisation de grande quantité de pesticides chimiques d'origine et de qualité diverses (Agbohessi, 2014) pour qui les écosystèmes aquatiques constituent les ultimes réceptacles (Chao et al., 2009) avec d'importants dégâts sur leur biodiversité (Agbohessi et al., 2015a).

Plusieurs études ont signalé des concentrations alarmantes de pesticides dans la rivière Alibori et dans les retenues d'eau situées dans le bassin cotonnier (Soclo et al., 2004; Yèhouénou A. Pazou, 2005 ; Adam et al., 2010 ; Gbaguidi, 2011; Agbohessi et al., 2012 ; Agbohessi et al., 2015a). Cependant, hormis les travaux de Agbohessi (2014), les impacts biologiques de ces molécules toxiques ont été très peu évalués sur les espèces aquatiques exploitées par les populations, notamment les poissons. C'est ce qui justifie la présente étude dont l'objectif principal est d'évaluer l'état de santé des poissons, notamment $O$. niloticus, élevé dans les retenues d'eau du bassin cotonnier (en happas et en enclos) à partir des biomarqueurs, notamment les indices biométriques et des paramètres de croissance. 


\section{MATERIEL ET METHODES Milieu d'étude}

L'étude a été menée en saison agricole dans deux retenues d'eau; il s'agit de la retenue de Batran (20 ha; Latitude = $3^{\circ} 52^{\prime} 18^{\prime \prime} \quad$ et Longitude $=9^{\circ} 49^{\prime} 55^{\prime \prime}$ ), considéré comme étant la plus exposée aux pesticides agricoles du fait de sa situation dans la commune la plus consommatrice de pesticides au Bénin (Banikoara); et de la retenue d'eau de Songhaï (41,5 ha ; Latitude = $4^{\circ} 12^{\prime} 56^{\prime \prime}$ et Longitude = 7०51'26"), considérée comme étant moins polluée du fait qu'elle soit située hors du bassin cotonnier (à environ $350 \mathrm{~km}$ au Sud de Banikoara, dans la commune de N'Dali). Ces deux retenues d'eau choisies sont fonctionnelles et ne tarissent pas en saison sèche. Elles sont dans la même zone agro-climatique et sont soumises au même climat de type soudanosoudanienne avec une seule saison de pluie allant de mai à septembre et une seule saison sèche allant d'octobre à avril (Adam et Boko, 1993).

\section{Matériel biologique}

L'espèce étudiée est Oreochromis niloticus (Figure 1), qui est la plus abondante dans les retenues d'eau du Nord Bénin (Imorou Toko et al., 2011). Elle appartient à la Famille des Cichlidae, à l'Ordre des Perciformes, à la Classe des Osteichthyens et à l'Embranchement des Vertébrés. C'est une espèce euryèce et disponible en toute saison, sensible à la pollution et présente dans toutes les retenues d'eau du Nord-Bénin.

\section{Approche et conduite expérimentales}

L'approche expérimentale in situ a été utilisée durant la présente étude. Pour ce faire, des juvéniles de $O$. niloticus (poids moyen initial 12,53 $\pm 0,44 \mathrm{~g}$ et de longueur totale moyenne $9,73 \pm 0,08 \mathrm{~cm}$ ) sexes mélangés ont été achetés au Centre Piscicole «Royal Fish Bénin » sis à Porto Novo et transportés dans des poches remplies d'oxygène à raison de 100 alevins par poche de 30 litres. Une fois sur le terrain, les alevins ont été acclimatés durant 10 jours dans des filets poches (happas) installés dans chaque retenue d'eau à raison de 200 alevins par happas de $8 \mathrm{~m}^{3}$.

Dans chaque retenue d'eau (Songhaï et Batran), le dispositif expérimental est composé de 3 happas ( 1 x 1 x 1,80 m chacun) et 3 enclos ( $1 \times 1 \times 3 \mathrm{~m}$ chacun). Les happas et les enclos sont des poches fabriquées au moyen de filet de pêche de $1 \mathrm{~cm}$ de maille, fixés chacun sur des tubes métalliques installés aux quatre côtés de chaque poche et solidement enfoncés dans la vase. Les happas se différencient des enclos par le fait qu'ils ne permettent pas aux poissons d'avoir accès au sédiment, tandis que dans les enclos les poissons ont accès au sédiment du fait que leur base repose sur la vase de fond.

Dans chaque happas et dans chaque enclos, la mise en charge a été faite avec 75 alevins de $O$. niloticus après la période d'acclimatation in situ. La biomasse initiale a été mesurée dans chaque poche, de même que la longueur totale individuelle de 30 alevins choisis au hasard par retenue d'eau avant leur répartition dans les happas et les enclos. En fin d'expérimentation, soit après 50 jours d'élevage, le nombre et la biomasse des alevins ont été mesurés dans chaque happas et enclos. La longueur totale individuelle de 10 poissons par happas et par enclos a été également mesurée en fin d'expérimentation.

Les alevins mis en charge ont été nourris à satiété apparente deux fois par jour ( $8 \mathrm{~h}$ et $16 \mathrm{~h}$ ) durant 50 jours. Les granulés commerciaux pour tilapia de marque « Le GOUESSANT, France » et titrant $9 \%$ de matières grasses brutes et $32 \%$ de protéines brutes ont été utilisés durant toute l'expérimentation. La quantité d'aliment distribuée dans chaque happas et dans chaque enclos a été enregistrée tous les jours.

La température de l'eau $\left({ }^{\circ} \mathrm{C}\right)$ de même que le pH ont été mesurés tous les jours (Multimètre de marque HANNA HI 9811-5 dans chaque retenue d'eau à $7 \mathrm{~h}$ et à $17 \mathrm{~h}$ aux abords du dispositif d'expérimentation. 


\section{Echantillonnage et conservation des échantillons}

Avant la mise en charge, le foie et les gonades ont été prélevés et pesés individuellement (balance électronique de marque KERN, de portée $1 \mathrm{~kg}$, sensible à 0,001 près) chez un lot de 10 mâles et 10 femelles choisi au hasard dans les happas d'acclimatation installés dans chaque retenue d'eau. Le poids individuel de chaque individu a été également mesuré (balance électronique de marque PHILIPS, de portée $5 \mathrm{~kg}$, sensible à 0,1 près) avant éviscération.

Après 50 jours d'élevage dans chaque milieu, le foie et les gonades ont été prélevés et pesés individuellement (balance électronique de marque KERN, de portée 1 $\mathrm{kg}$, sensible à 0,001 près) sur un échantillon aléatoire de 10 mâles et 10 femelles par happas et par enclos. Le poids individuel de chaque individu a été également mesuré (balance électronique de marque PHILIPS, de portée $5 \mathrm{~kg}$, sensible à 0,1 près) avant dissection.

Afin de comparer les données recueillies chez les poissons élevés en happas et en enclos à celles des poissons existants dans le milieu naturel, les mêmes organes ont été prélevés et les mêmes données mesurées de la même façon chez un échantillon de 10 mâles et 10 femelles de $O$. niloticus capturés en eau libre dans chaque retenue d'eau en fin d'expérience. Pour chaque échantillonnage, les poissons ont été anesthésiés $(180 \mathrm{mg}$ du MS 222 par litre d'eau), puis euthanasiés par dislocation cervicale.

\section{Paramètres calculés}

Des paramètres de croissance de même que des indices biométriques ont été calculés à partir des différentes données prélevées chez les poissons aussi bien à Songhaï qu'à Batran.

Paramètres de survie et de croissance des poissons élevés en happas et en enclos

○ Taux de survie (TS): TS $(\%)=$ Nombre final x 100/Nombre initial
○ Poids moyen final (Pmf): Pmf (g) = Biomasse finale $(\mathrm{g}) /$ Nombre final

○ Gain de poids moyen (GP): GP (g) = Poids moyen final (g) - Poids moyen initial $(\mathrm{g})$ avec Poids moyen initial $($ Pmi $)=$ Biomasse initial (g)/Effectif initial

- Taux de croissance spécifique (TCS): TCS (\%/jour $)=[(\ln$ Pmf $-\ln$ Pmi $) /$ durée d'élevage (jour)] x 100

\section{Indices biométriques}

- Indice de condition $(\mathrm{K}): \mathrm{K}(\%)=\left(\mathrm{P} / \mathrm{LT}^{3}\right)$ $\mathrm{x} 100$; avec P : Poids corporel $(\mathrm{g})$ et LT : Longueur totale $(\mathrm{cm})$

- Indice hépato-somatique (IHS) : IHS (\%) $=(\mathrm{Pf} / \mathrm{P}) \times 100 ;$ avec Pf : Poids du foie $(\mathrm{g})$ et $\mathrm{P}(\mathrm{g})$ : Poids corporel

- Indice gonado-somatique (IGS) : IGS (\%) $=(\mathrm{Pg} / \mathrm{P}) \times 100 ;$ avec $\mathrm{Pg}:$ Poids des gonades $(\mathrm{g})$ et $\mathrm{P}(\mathrm{g})$ : Poids corporel

Aussi bien pour les paramètres de survie, de croissance que les indices biométriques, les valeurs calculées sont présentées sous forme de moyenne plus ou moins erreur-standard.

\section{Analyses statistiques des données}

Les données ont été saisies dans le tableur Excel 2007 et traitées grâce au logiciel statistique Minitab 16. Le test de Ryan-Joiner et le test de Levene ont été utilisés pour apprécier respectivement la normalité et l'homoscédasticité des données. Pour des comparaisons deux à deux des moyennes, le test $\mathrm{T}$ de Student à deux échantillons indépendants a été utilisé. L'Analyse de variance à un critère de classification (ANOVA 1) a été utilisée pour comparer les moyennes relatives aux paramètres de croissance, de même que les indices biométriques en eau libre, en enclos et en happas. Les données biométriques ont été analysées par sexe et par type d'infrastructure (happas et enclos), tandis que les paramètres de croissance et de survie ont été comparés par type d'infrastructure en fonction du milieu. Pour toutes ces analyses, le seuil de significativité était de $5 \%$. 


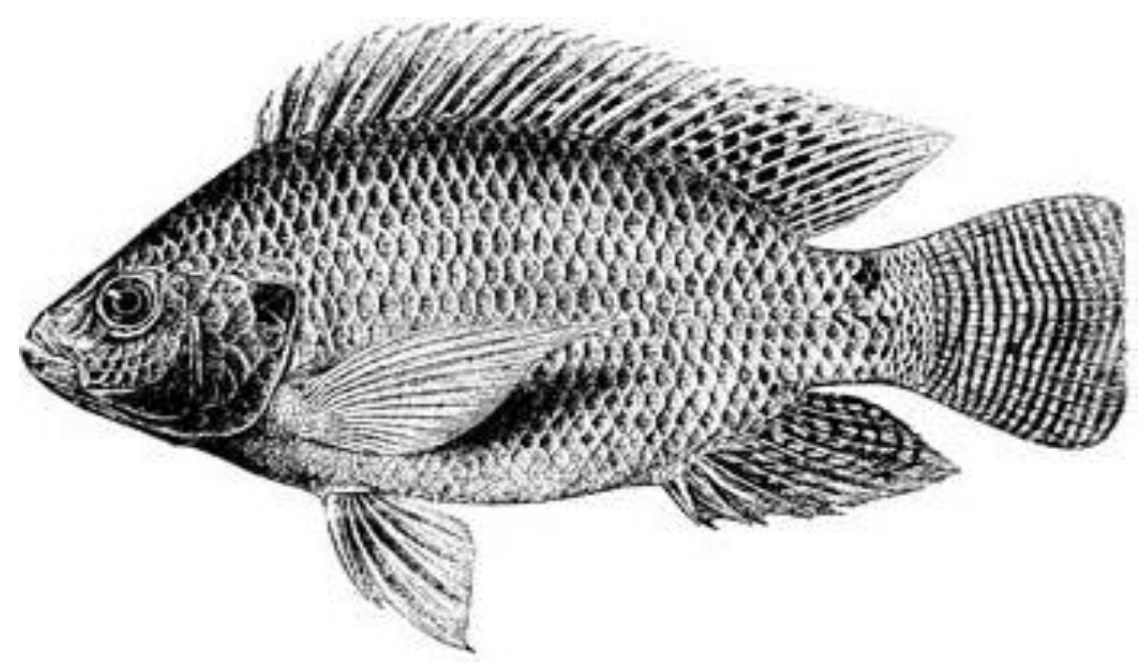

Figure 1: Schéma d'un spécimen de Oreochromis niloticus (Linnaeus, 1758).

\section{RESULTATS}

Qualité des eaux dans les retenues d'eau

Les valeurs moyennes de température $\left(28,51 \pm 0,16{ }^{\circ} \mathrm{C}\right.$ et $28,26 \pm 0,99{ }^{\circ} \mathrm{C}$, respectivement à Batran et à Songhaï) et de $\mathrm{pH}(6,9 \pm 0,6$ et $6,81 \pm 0,13$, respectivement à Batran et à Songhaï) enregistrées durant la présente étude ne sont pas significativement différentes d'une retenue d'eau à l'autre $(\mathrm{p}>0,05)$.

\section{Paramètres zootechniques en happas et en enclos}

Les paramètres de survie et de croissance mesurés chez les poissons élevés dans les happas et les enclos installés dans les deux retenues d'eau sont présentés dans le Tableau 1.

Les taux de survie des poissons maintenues dans les happas $(75,56 \pm 8,88 \%$ et $91,56 \pm 0,77 \%$ respectivement à Songhaï et à Batran) ont été meilleurs $(\mathrm{p}<0,05)$ à ceux enregistrés chez les poissons élevés en enclos $(56,44 \pm 34,70 \%$ à Songhaï et $87,11 \pm 4,68 \%$ à Batran). On note également de meilleurs taux de survie chez les poissons élevés à Batran qu'à Songhaï aussi bien en enclos qu'en happas $(p<0,05)$. Cependant, il est important de signaler que dans la retenue d'eau de Songhaï, les taux de survie enregistrés ne sont pas dus aux mortalités des poissons, mais surtout à des fuites de poissons consécutives aux fortes pluies qui ont engendré une monté brusque du niveau de l'eau dans la retenue, allant jusqu'à engloutir certains enclos, durant le mois de juillet.

Contrairement à la survie, la croissance (Pmf et GP) des poissons a été meilleure à Songhaï qu'à Batran $(\mathrm{p}<0,05)$. A Songhaï, le poids moyen final $(96,67 \pm 0,9 \mathrm{~g})$ de même que le gain de poids $(84,15 \pm 0,95 \mathrm{~g})$ des poissons maintenus en happas sont significativement meilleurs à ceux enregistrés dans les enclos $(\mathrm{Pmf}=89,44 \pm 0,7 \mathrm{~g}$ et $\mathrm{GP}=$ $76,89 \pm 1,7 \mathrm{~g}$ ); tandis que le contraire est observé à Batran. Cette même tendance est observée pour le taux de croissance spécifique bien que les différences observées ne soient pas significatives (Figure 2).

Indices biométriques de $\boldsymbol{O}$. niloticus dans les retenues d'eau de Songhaï et de Batran

Le Tableau 2 présente les indices biométriques (IGS, IHS et K) de $O$. niloticus en eau libre, en enclos et en happas, respectivement dans les retenues d'eau de Songhaï et de Batran.

\section{Indice gonado-somatique (IGS)}

Chez les mâles, l'IGS n'a pas révélé de différences significatives $(p>0,05)$ entre les 
poissons élevés en enclos et en happas, aussi bien à Songhaï $(0,63 \pm 0,0 \%$ et $0,74 \pm 0,04 \%$ respectivement en enclos et en happas) qu'à Batran $(0,62 \pm 0,11 \%$ et $0,63 \pm 0,07 \%$ respectivement en enclos et en happas) (Figures 3 et 4). Cependant dans les deux milieux, on a observé chez les mâles capturés en eau libre des IGS plus faibles $(\mathrm{p}<0,05)$ que ceux obtenus chez les poissons élevés dans les infrastructures (enclos et happas). Chez les femelles, les IGS des poissons élevés en happas $(4,10 \pm 0,01 \%$ à Songhaï et $4,25 \pm$ $0,33 \%$ à Batran) sont plus élevés $(\mathrm{p}<0,05)$ que ceux obtenus chez les poissons maintenus en enclos $(1,86 \pm 0,05 \%$ à Songhaï et 2,29 \pm $0,61 \%$ à Batran) (Figures 3 et 4 ). A Songhaï on n'observe pas de différences significatives entre l'IGS des femelles élevées en enclos comparativement à celles capturées en eau libre ; cependant à Batran, cette différence s'est révélée significative avec des IGS plus faibles obtenus chez les femelles capturées en eau libre.

\section{Indice hépato-somatique (IHS)}

Comme pour l'IGS, l'IHS chez les mâles n'a pas montré de différences significatives entre les poissons élevés en enclos et en happas dans les deux milieux (Figures 3 et 4). Cependant, les IHS des poissons élevés dans les infrastructures (enclos et happas) sont plus élevés $(p<0,05)$ que ceux obtenus chez les poissons capturés en eau libre dans les deux retenues d'eau.

La même tendance observée chez les mâles a été observée chez les femelles dans la retenue d'eau de Batran (Figure 4). Cependant à Songhaï (Figure 3), des différences significatives ont été observées avec des IHS plus élevés obtenus chez les poissons élevés en happas et plus faibles chez ceux capturés en eau libre.

\section{Indice de condition $(K)$}

Chez les mâles, l'indice de condition a varié de la même manière que l'IGS et l'IHS aussi bien à Songhaï qu'à Batran (Figures 3 et 4). On observe donc pas de différences significatives entre les poissons élevés en enclos et ceux en happas ( $p>0,05$ ), qui présentent par contre des indices de condition meilleurs $(\mathrm{p}<0,05)$ à ceux capturés en eau libre dans les deux retenues d'eau. Ces mêmes tendances ont été observées chez les femelles aussi bien à Songhaï qu'à Batran (Figures 3 et 4).

Tableau 1: Synthèse des paramètres zootechniques mesurés chez les poissons élevés dans les happas et dans les enclos à Songhaï (témoin) et à Batran (contaminée).

\begin{tabular}{|c|c|c|c|c|}
\hline \multirow{2}{*}{ Paramètres } & \multicolumn{2}{|c|}{ Retenue d'eau de Songhaï } & \multicolumn{2}{|c|}{ Retenue d'eau de Batran } \\
\hline & Enclos & Happas & Enclos & Happas \\
\hline Taux de survie (\%) & $56,44 \pm 34,70^{a}$ & $75,56 \pm 8,88^{b}$ & $87,11 \pm 4,68^{\mathrm{c}}$ & $91,56 \pm 0,77^{d}$ \\
\hline Pmi (g) & $12,55 \pm 0,47^{\mathbf{a}}$ & $12,52 \pm 0,42^{a}$ & $12,51 \pm 0,41^{\mathrm{a}}$ & $12,54 \pm 0,46^{\mathbf{a}}$ \\
\hline $\operatorname{Pmf}(g)$ & $89,44 \pm 0,7^{\mathbf{a}}$ & $96,67 \pm 0,9^{\mathbf{b}}$ & $80,2 \pm 0,92^{\mathbf{c}}$ & $71,85 \pm 0,69^{d}$ \\
\hline GP $(g)$ & $76,89 \pm 1,7^{\mathrm{a}}$ & $84,15 \pm 0,95^{\mathbf{b}}$ & $67,69 \pm 1,3^{\mathrm{c}}$ & $59,31 \pm 1,1^{\mathbf{d}}$ \\
\hline
\end{tabular}

Les valeurs d'une même ligne ayant des lettres différentes en exposant sont significativement différentes au seuil de 5\%. 


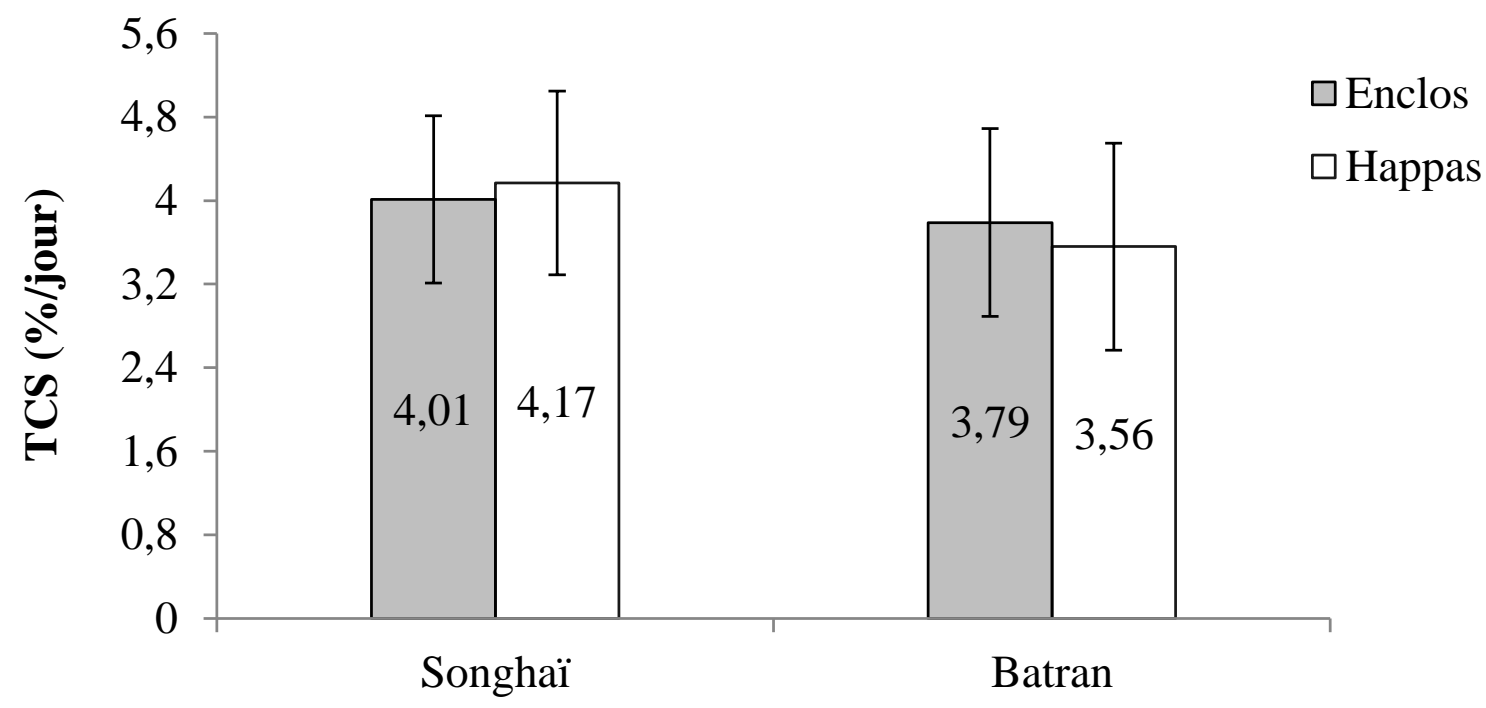

\section{Retenue d'eau}

Figure 2: Taux de croissance spécifique (TCS) de $O$. niloticus élevés en happas et en enclos dans les retenues d'eau témoin de Songhaï et contaminée de Batran.

Tableau 2: Indices biométriques (IGS, IHS et K) de O. niloticus en eau libre, en enclos et en happas dans les retenues d'eau témoin de Songhaï et contaminée de Batran.

\begin{tabular}{|c|c|c|c|c|c|c|}
\hline \multirow[b]{2}{*}{ Indices } & \multirow{2}{*}{\multicolumn{2}{|c|}{ Infrastructure }} & \multicolumn{2}{|c|}{ Mâles } & \multicolumn{2}{|c|}{ Femelles } \\
\hline & & & Songhaï & Batran & Songhaï & Batran \\
\hline \multirow{4}{*}{$\operatorname{IGS}(\%)$} & Eau libre & & $0,35 \pm 0,61^{\mathrm{a}}$ & $0,27 \pm 0,03^{b}$ & $1,80 \pm 0,01^{\mathrm{a}}$ & $0,96 \pm 0,15^{b}$ \\
\hline & Enclos & & $0,63 \pm 0,05^{\mathbf{a}}$ & $0,62 \pm 0,11^{\mathbf{a}}$ & $1,86 \pm 0,05^{\mathrm{a}}$ & $2,29 \pm 0,61^{\mathbf{b}}$ \\
\hline & Happas & & $0,74 \pm 0,04^{\mathbf{a}}$ & $0,63 \pm 0,07^{\mathbf{a}}$ & $4,10 \pm 0,01^{\mathrm{a}}$ & $4,25 \pm 0,33^{\mathbf{a}}$ \\
\hline & & $p$ & 0,000 & 0,001 & 0,000 & 0,000 \\
\hline \multirow{4}{*}{$\operatorname{IHS}(\%)$} & Eau libre & & $0,31 \pm 0,08^{\mathbf{a}}$ & $0,19 \pm 0,01^{\mathbf{a}}$ & $0,62 \pm 0,02^{\mathrm{a}}$ & $0,59 \pm 0,11^{\mathbf{a}}$ \\
\hline & Enclos & & $2,94 \pm 0,2^{\mathrm{a}}$ & $2,60 \pm 0,21^{\mathbf{a}}$ & $1,77 \pm 0,08^{\mathbf{a}}$ & $2,53 \pm 0,22^{\mathbf{b}}$ \\
\hline & Happas & & $2,96 \pm 0,22^{a}$ & $3,06 \pm 0,2^{\mathbf{a}}$ & $2,90 \pm 0,06^{\mathbf{a}}$ & $2,67 \pm 0,2^{\mathbf{a}}$ \\
\hline & & $p$ & 0,000 & 0,000 & 0,000 & 0,000 \\
\hline \multirow{4}{*}{$\mathrm{K}(\%)$} & Eau libre & & $1,04 \pm 0,02^{\mathrm{a}}$ & $1,03 \pm 0,01^{\mathrm{a}}$ & $1,99 \pm 0,05^{\mathrm{a}}$ & $1,66 \pm 0,05^{b}$ \\
\hline & Enclos & & $1,79 \pm 0,05^{\mathrm{a}}$ & $1,86 \pm 0,04^{\mathrm{a}}$ & $2,76 \pm 0,02^{\mathbf{a}}$ & $1,99 \pm 0,04^{\mathbf{b}}$ \\
\hline & Happas & & $1,98 \pm 0,1^{\mathbf{a}}$ & $1,81 \pm 0,05^{\mathrm{a}}$ & $2,44 \pm 0,04^{\mathbf{a}}$ & $1,98 \pm 0,02^{\mathbf{b}}$ \\
\hline & & $p$ & 0,002 & 0,007 & 0,008 & 0,000 \\
\hline
\end{tabular}

Les valeurs d'une même ligne (en fonction du sexe) ayant des lettres différentes en exposant sont significativement différentes au seuil de 5\% pour le test $T$ de Student. Les valeurs de la probabilité p du tableau sont celles obtenues de ANOVA 1 et sont à considérer pour les Figures 3 et 4. 


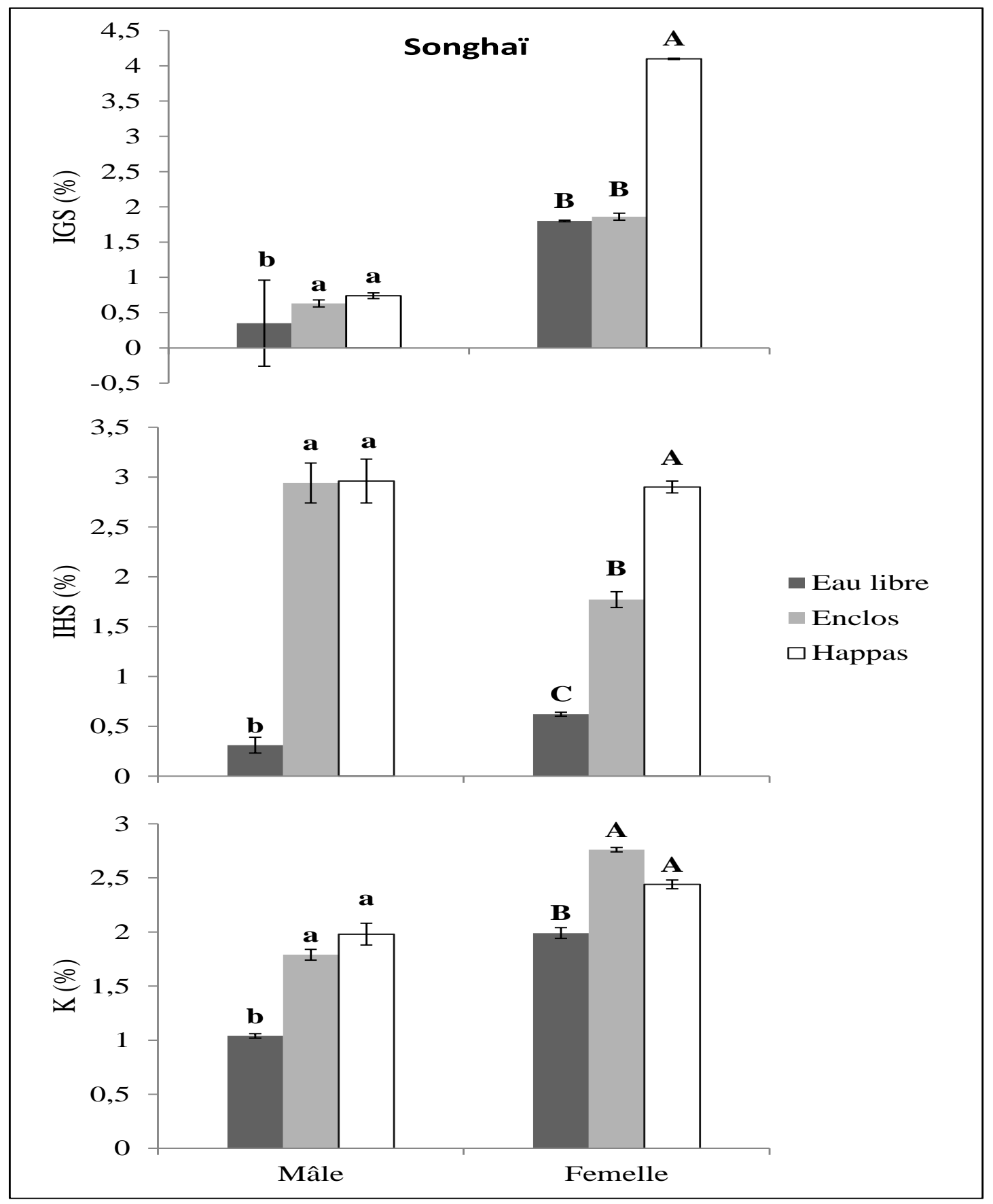

Figure 3: L'indice gonado-somatique (IGS), l'indice hépato-somatique (IHS) et l'indice de condition (K) des mâles et des femelles de $O$. niloticus capturés en eau libre, en enclos et en happas dans la retenue d'eau témoin de Songhaï (Les histogrammes d'un même sexe portants des lettres différentes (a et $b$ pour les mâles et $A, B$ et $C$ pour les femelles) sont significativement différentes au seuil de 5\%). 


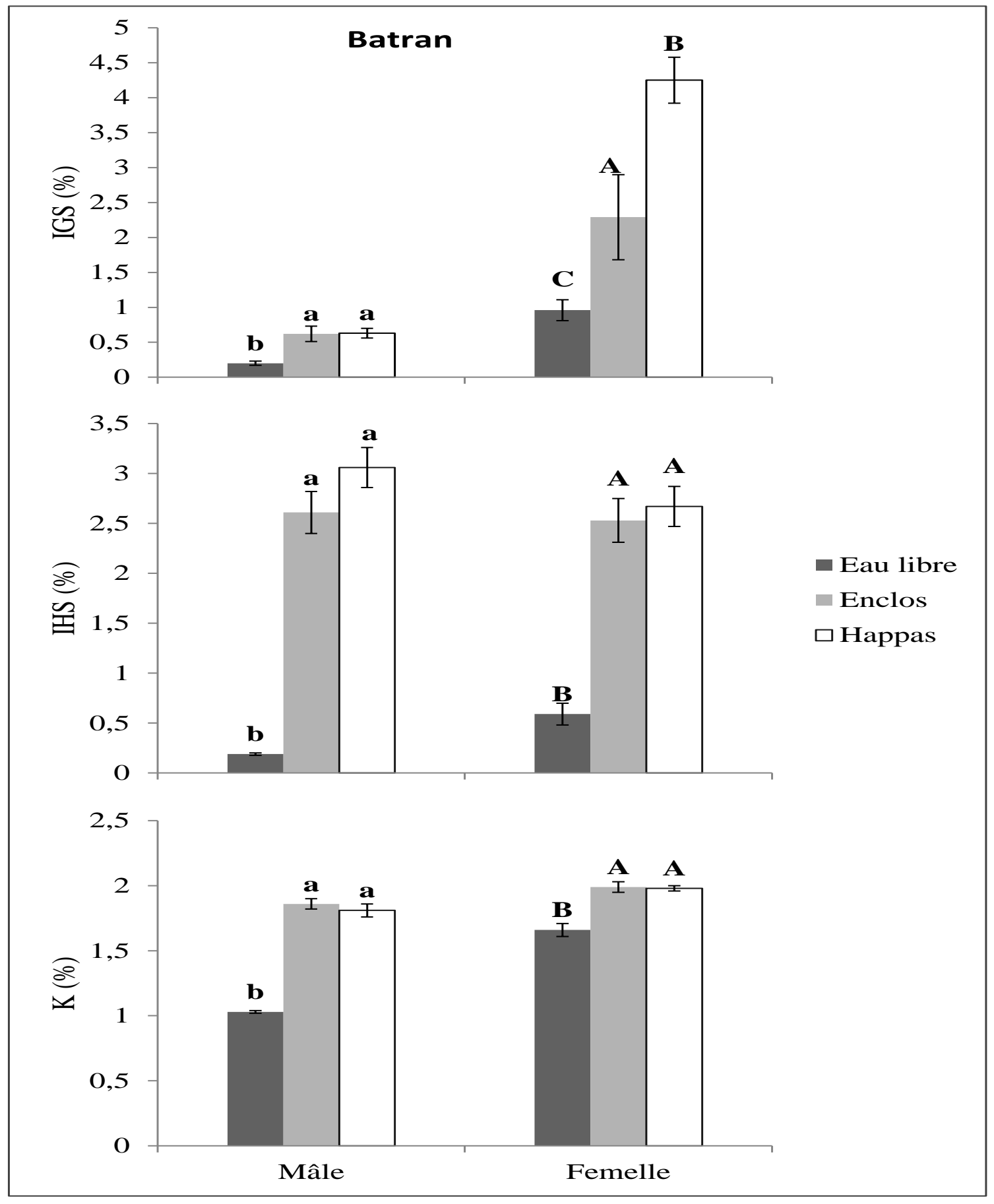

Figures 4: L'indice gonado-somatique (IGS), l'indice hépato-somatique (IHS) et l'indice de condition (K) des mâles et des femelles de O. niloticus capturés en eau libre, en enclos et en happas dans la retenue d'eau de Batran (Les histogrammes d'un même sexe portants des lettres différentes (a et $b$ pour les mâles et $A, B$ et $C$ pour les femelles) sont significativement différentes au seuil de $5 \%$. 


\section{DISCUSSION}

Durant la présente étude, les valeurs de température et de $\mathrm{pH}$ enregistrées dans les deux retenues d'eau sont dans les normes tolérées par les espèces tropicales d'eau douce dont le tilapia $O$. niloticus (Malcolm et al., 2000 ; Lacroix, 2004 ). Elles sont très proches des valeurs mesurées par Imorou Toko et al. (2011) dans les retenues d'eau de la commune de Banikoara, et des valeurs signalées par Chabi et al. (2012) dans les retenues d'eau du département du Borgou (Nord-Bénin), notamment celles des communes de Tchaourou, Nikki, Péréré, N'Dali et Parakou.

En dépit des dégâts liés à l'inondation survenue dans la retenue d'eau de Songhaï, les taux de survie de $O$. niloticus obtenus dans les deux retenues d'eau sont assez bonnes ( $>50 \%$ ) et augurent bien de la qualité de leurs eaux à maintenir en vie une espèce aussi exigeante. Les valeurs de survie obtenues aussi bien en enclos qu'en happas sont d'une part, proches de celles rapportées par Elègbè et al. (2015a , 2015b) dans les whedos du haut delta de l'Ouémé au Bénin, et d'autre part, de celles enregistrées par Iga-Iga (2008) en cages flottantes au Gabon et Sarr et al. (2015) en étangs piscicoles au Sénégal pour $O$. niloticus.

Les performances de croissance (poids moyen final, gain de poids et taux de croissance spécifique) enregistrées durant la présente étude aussi bien à Songhaï qu’à Batran sont supérieures à celles obtenues par Iga-Iga (2008) qui a testé plusieurs régimes de fabrication locale chez $O$. niloticus maintenu en cages en milieu lagunaire. Les meilleures performances de croissance observées chez les poissons élevés dans la retenue d'eau de Songhaï comparativement à celle de Batran (en enclos comme en happas) pourraient bien refléter l'état de santé des poissons dans ces deux milieux. En effet, pour la durée de l'essai, la différence de croissance des poissons dans ces deux retenues résulterait de la diminution de l'efficacité d'utilisation des aliments, induite notamment par la présence de résidus de pesticides agricoles, dans la retenue de Batran (Agbohessi et al., 2014 ; Agbohessi et al., 2015a).
Bien qu'il soit quasi impossible de trouver dans le Nord du Bénin des écosystèmes aquatiques encore exempts de pesticides, il est tout de même trivial que la retenue d'eau de Batran située dans le bassin cotonnier béninois reçoit plus d'effluents contenant des résidus de pesticides chimiques coton que celle de Songhaï située dans une zone à plus faible usage de pesticides coton. Ceci pourrait donc justifier qu'aussi bien chez les mâles que chez les femelles, les indices biométriques (IGS, IHS et K) de O. niloticus capturés en eau libre soient plus élevés dans la retenue d'eau de Songhaï que dans celle de Batran.

En effet, plusieurs études ont révélé que l'IGS des poissons dans des milieux pollués sont faibles comparativement à ceux des poissons capturés dans des milieux moins pollués. C'est le cas par exemple du Tilapia guineensis capturé dans diverses stations de la rivière Alibori (Agbohessi et al., 2015a) et de Clarias gariepinus exposé à différentes doses de Tihan (175 O Teq) et d'endosulfan (Agbohessi et al., 2015b). Chez toutes ces espèces, la diminution de l'indice gonadosomatique s'explique par la présence des altérations régressives dans les gonades qui contribuent à la diminution de leur poids et par la diminution des réserves vitellogeniques comme l'ont montré Agbohessi et al. (2015a , 2015b) et Oreins (2016).

Chez les poissons, la diminution de l'IHS témoigne par contre d'une atteinte du foie par des polluants chimiques comme les pesticides. La majorité des pesticides appliqués par les cotonculteurs dans la zone d'étude présente un coefficient de partition octanol-eau (Kow) élevé, ce qui suggère leur haut potentiel de bioconcentration dans les organes riches en lipides comme le foie (Soderlund et al., 2002). En effet, la diminution de l'IHS serait liée à une diminution de réserves glycogéniques utilisées pour lutter contre le stress chimique et à une forte présence d'altérations régressives dans le foie (Agbohessi et al., 2015a, 2015b). Belhamra (2012) a également rapporté une diminution de l'IHS après exposition du 
poisson Gambusia affinis à l'organophosphoré Actara. Cependant, Agbohessi et al. (2015b) ont observé une augmentation de l'IHS chez Clarias gariepinus exposé au Tihan (175 O Teq) en condition de laboratoire. Selon ces auteurs, l'augmentation de l'IHS s'expliquerait par l'augmentation du foie attribuable à l'hyperplasie et à l'hypertrophie des cellules hépatiques, une réponse jugée adaptative augmentant ainsi l'activité des enzymes biotransformatrices et de détoxification du foie.

Les faibles indices de condition $(\mathrm{K})$ obtenus dans la retenue de Batran comparativement à celle de Songhaï pourraient aussi être liés au degré de pollution en pesticides dans les deux milieux. Selon Laflamme et al. (2000), l'indice de condition du poisson est négativement affecté par la contamination environnementale. Marchand et al. (2009) rapportent également que la présence des pesticides dans le milieu affecte négativement la prise alimentaire et diminue les ressources alimentaires (plancton et macroinvertébrés benthiques) disponibles aux poissons. Ainsi, même si le poisson se nourrit, l'énergie serait donc prioritairement utilisée pour activer les mécanismes de lutte contre les polluants au détriment de la croissance (Agbohessi et al., 2015b).

Dans les deux retenues d'eau, les indices biométriques (IGS, IHS et $\mathrm{K}$ ) des poissons capturés directement en eau libre sont généralement significativement plus faibles que ceux obtenus chez les poissons élevés en enclos ou en happas. Ceci pourrait être lié à la durée d'exposition aux polluants relativement courte des poissons élevés en enclos et en happas (50 jours), comparativement à ceux capturés en eau libre où ils vivent depuis plusieurs générations. En effet, selon Camiré (2007), la chronicité et la durée de l'exposition des poissons aux pesticides même à de faibles doses pourraient expliquer les dommages biologiques, physiologiques et morphologiques observés.

Par ailleurs, les différences observées entre l'IGS et l'IHS des poissons (notamment les femelles) élevés en enclos et en happas seraient liées aux caractéristiques de ces infrastructures d'élevage. En effet, contrairement aux happas, les enclos offrent la possibilité aux poissons qui y sont élevés d'avoir accès aux sédiments où sont généralement piégés les molécules de même que leurs dérivés les plus toxiques qui polluent les milieux aquatiques (Yèhouénou et Pazou, 2005 ; Adam et al., 2010 ; Agbohessi, 2014). Or, la majorité des insecticides et herbicides utilisés dans le bassin cotonnier ont un coefficient de partage carbone organique-eau (Koc) élevé (Agbohessi, 2014). Ceci sous-tend leur grande affinité pour les sédiments, donc ont d'effets plus prononcés sur les organismes proches ou en contact avec ces sédiments (Fojut et al., 2012). La lambdacyhalothrine (pyréthrinoïde) et l'acétamipride (néonicotinoïde), principales matières actives des pesticides du bassin cotonnier (Zoumenou et al., 2015) se fixent aux sédiments et se dégradent moins vite que celles dissoutes dans la colonne d'eau puisqu'ils sont moins accessibles à la dégradation solaire. En enclos, la proximité de $O$. niloticus des sédiments pourrait donc justifier les faibles valeurs d'IGS, d'IHS et de K observés aussi bien chez les mâles que chez les femelles à Songhaï comme à Batran.

\section{Conclusion}

Il ressort de la présente étude que les performances de survie et de croissance des alevins de Oreochromis niloticus élevés dans les retenues d'eau du Nord Bénin sont assez bonnes, et ceux malgré les qualités physicochimique et sanitaire des eaux de ces milieux. Cependant, les indices biométriques, notamment l'indice gonado-somatique, l'indice hépato-somatique et l'indice de condition des poissons élevés dans les retenues d'eau de Songhaï et de Batran (surtout ceux capturés en eau libre) révèlent qu'aussi bien chez les mâles que les femelles, les poissons à Batran sont plus affectés par la qualité des eaux (probablement plus exposées aux pesticides chimiques agricoles) de cette retenue. Chez les femelles à Songhaï comme à Batran, les indices biométriques sont 
généralement meilleurs en happas comparativement aux enclos qui exposent plus les poissons aux pesticides piégés dans la vase. La présente étude sera davantage renforcée par les résultats des analyses physico-chimiques des eaux des deux retenues (notamment le dosage des résidus de pesticides dans différentes matrices) de même que les résultats des analyses d'autres biomarqueurs aux niveaux biochimique, histologique et physiologique qui sont en cours sur les échantillons d'organes prélevés lors des échantillonnages.

\section{CONFLIT D'INTERETS}

Les auteurs déclarent qu'il n'y a aucun conflit d'intérêts pour cet article.

\section{CONTRIBUTIONS DES AUTEURS}

IIT a identifié la thématique de la recherche, élaboré le protocole, supervisé cette étude et rédigé le manuscrit. ROEP a effectué son mémoire de master sur cette thématique et a donc suivi les expérimentations sur le terrain et contribué à la rédaction de la première version de ce manuscrit. RT était stagiaire au LaRAEAq et a participé activement au déroulement des expérimentations. LNG est une doctorante qui a également dirigé les opérations de collecte des données sur le terrain et a corrigé les premières versions de ce manuscrit. PTA est le directeur scientifique du projet AquaTox et a corrigé le protocole et le manuscrit. PK est le coordonnateur belge du projet AquaTox et a contribué à ce titre à la conception des objectifs et hypothèses de cette recherche.

\section{REMERCIEMENTS}

Les auteurs expriment leur reconnaissance à l'endroit du Comité de gestion de la retenue d'eau de Batran, de même que tous les pêcheurs de ce village qui ont contribué à l'installation des infrastructures expérimentaux et aux pêches. Nous remercions également les responsables du Centre Songhaï de Atagara qui nous ont permis de mener gracieusement ces recherches dans leur retenue d'eau.

\section{REFERENCES}

Adam KS, Boko M. 1993. Le Bénin (2ème édition). EDICEF: Paris; p.96.

Adam S, Edorh PA, Totin H, Koumolou L, Amoussou E, Aklikokou K, Boko M. 2010. Pesticides et métaux lourds dans l'eau de boisson, les sols et les sédiments de la ceinture cotonnière de Gogounou, Kandi et Banikoara (Bénin). International Journal of Biological and Chemical Sciences, 4(4): 1170-1179. DOI:

http://dx.doi.org/10.4314/ijbcs.v4i4.6305 4

Adam Sanni M. 2011. Valorisation piscicole des retenues d'eau de la commune de Banikoara: Potentialités et limites. Thèse d'ingénieur agronome, Faculté d'Agronomie, l'Université de Parakou, Bénin, p. 81.

Adechian SA, Baco MN, Akponikpe I, Imorou Toko I, Egah J, Affoukou K. 2015. Les pratiques paysannes de gestion des pesticides sur le maïs et le coton dans le bassin cotonnier du Bénin. Temporalités, Action Environnementale et Mobilisations Sociales, 15(2). DOI: 10.4000/vertigo. 16534

Agbohessi TP, Imorou Toko I, Kestemont P. 2012. Etat des lieux de la contamination des écosystèmes aquatiques par les pesticides organochlorés dans le bassin cotonnier béninois.Cahiers Agricultures, 21(1): 46-56. DOI: https://doi.org/10.1684/agr.2012.0535

Agbohessi TP, Imorou Toko I, N'tcha I, Geay F, Mandiki SNM, Kestemont P. 2014. Exposure to agricultural pesticides impairs growth, feed utilization and energy budget in African Catfish Clarias gariepinus (Burchell, 1822) fingerlings. International Aquatic Research, 6(4): 229 - 243. DOI: 10.1007/s40071-0140083-5

Agbohessi TP. 2014. Impact des pesticides agricoles sur le développement et la régulation du système reproducteur, le statut hépatique et la croissance des poissons dans le bassin cotonnier béninois. Thèse de doctorat, Faculté des 
Sciences, Université de Namur, Belgique, p.307.

Agbohessi TP, Imorou Toko I, Ouédraogo A, Jauniaux T, Mandiki SNM, Kestemont P. 2015a. Assessment of the health status of wild fish inhabiting a cotton basin heavily impacted by pesticides in Benin (West Africa). Science of the Total Environment, 506-507: 567-584. DOI: 10.1016/j.scitotenv.2014.11.047

Agbohessi TP, Imorou Toko I, Atchou V, Tonato R, Mandiki SNM, Kestemont P. 2015b. Pesticides used in cotton production affect reproductive development, endocrine regulation, liver status and offspring fitness in African catfish Clarias gariepinus (Burchell, 1822). Comparative Biochemistry and Physiology, 167: DOI:10.1016/j.cbpc.2014.10.002

Al Dilaimi A. 2009. Détermination de la ration lipidique alimentaire optimale $\mathrm{du}$ tilapia du Nil (Oreochromis niloticus). Mémoire de master, Faculté des Sciences, Université d'Oran, Algérie, p.78.

Belhamra R. 2012. Activité d'un insecticide organophosphoré (Actara): Impact sur le système de détoxification, la croissance et la reproduction de Gambusia affinis. Mémoire de master, Faculté des Sciences, Université Badji MokhtarAnnaba, Algérie, p. 64.

Camiré M. 2007. Effets de l'exposition chronique aux pesticides sur le statut physiologique du poisson d'eau douce. Mémoire de maitrise, Faculté de Biologie, Université du Québec à Montréal, Canada, p. 82.

Chabi S, Imorou Toko I, Attakpa EY, Yabi JA, Elegbe H. 2012. Potentialités socioéconomiques et piscicoles des retenues d'eau du Borgou (nord Bénin). Communication orale. XV Edition des Journées Scientifiques Internationales de Lomé (JSIL), Université de Lomé, Lomé (Togo) du 22 au 26 Octobre.

Chao W, Guanghua L, Jing C, Perfang W. 2009. Sub-lethal effects of pesticide mixtures on selected biomarkers of Carassius auratus. Environmental Toxicology and Pharmacology, $\quad \mathbf{2 8 ( 3 ) :}$
414-419.

DOI: https://doi.org/10.1016/j.etap.2009.07.00 5

Elègbè AH, Imorou Toko I, Agbohessi P, Blé C, Banag A, Chikou A, Eyango Tomedi $\mathrm{M}$, Lalèyè $\mathrm{P}$. 2015a. Co-culture Clarias gariepinus-Oreochromis niloticus : quels avantages pour l'amélioration des performances zootechniques et économiques des poissons élevés dans les «whedos » du delta de l'Ouémé au Bénin? International Journal of Biological and Chemical Sciences, 9(4): $\quad$ 1937-1949. DOI: http://dx.doi.org/10.4314/ijbcs.v9i4.19

Elègbè AH, Agbohessi TP, Nkoué Békima $\mathrm{P}$, Imorou Toko I, Chikou A, Blé C, Lalèyè P, Eyango Tomedi M. 2015b. Effet du jeûne chez les juvéniles d'Oreochromis niloticus et de Clarias gariepinus sur la productivité des "whedos" du delta de l'Ouémé, Bénin, Afrique de l'Ouest. Afrique Science, 11(6): 125-138.

FAO (Organisation des Nations Unies pour l'alimentation et l'agriculture), 2016. La situation mondiale des pêches et de l'aquaculture 2016. Contribuer à la sécurité alimentaire et à la nutrition de tous. Rome, p. 224.

FAO (Organisation des Nations Unies pour l'alimentation et l'agriculture). 2010. La situation mondiale des pêches et de l'aquaculture 2010. Rome, p. 244.

Fojut TL, Palumbo AJ, Tjeerdema RS. 2012. Aquatic life water quality criteria derived via the UC Davis method: II. Pyrethroid Insecticides. Reviews of Environmental Contamination and Toxicology, 216: 51103. DOI: $10.1007 / 978-1-4614-2260-$ 0_2

Gbaguidi MMAN. 2011. Etude de la distribution et des impacts environnementaux et sanitaires des pesticides en zones de culture cotonnière au Bénin : Cas des bassins versants de la rivière Agbado et des plans d'eau des réserves de biosphère de la Pendjari et du W du Niger. Thèse de doctorat, Ecole Polytechnique d'Abomey-Calavi, Université d'Abomey-Calavi, p. 243.

Iga-Iga R. 2008. Contribution à la mise au point d'aliments pour tilapia 
Oreochromis niloticus à base d'intrants locaux : cas du Gabon. Mémoire de master, Institut de Recherches Agronomiques et Forestières, Gabon, p. 47.

Imorou Toko I, Yabi JA., Assogba MN., Adam Sanni M, Elègbè HA. 2011. Evaluation des potentialités piscicoles et socioéconomiques des retenus d'eau pastorales dans la commune de Banikoara (Nord- est du Bénin). Annales de l'Université de Parakou Série Sciences naturelles-Agronomie: 92 119.

Lacroix E. 2004. Pisciculture en Zone Tropicale. GFA Terra Systems -GTZ: Hamburg, p.225.

La flamme LS, Couillard Y, Campbell PGC, Hontela A. 2000. Interrenal metallothionein and cortisol secretion in relation to $\mathrm{Cd}, \mathrm{Cu}$, and $\mathrm{Zn}$ exposure in yellow perch, Perca flavescens, from Abitibi lakes. Canadian Journal of Fisheries and Aquatic Sciences, 57(8): 1692-1700.

Malcolm C, Beveridje H, Mc Andrew BJ. 2000. Tilapias: Biology and Exploitation. Institute of Aquaculture. University of Stirling, Scotland. Kluwer academic publishers, p. 185.

Marchand MJ. 2009. A histology-based fish health assessment to determine the health status and edibility of two indicator fish species from Roodeplaat Dam. Minor dissertation of Scientiae magister degree, Faculty of Science, University of Johannesburg, South Africa, p. 80.

Oreins N. 2016. Perturbations endocrines induites par l'Acer $35 \mathrm{EC}$, un insecticide à base d'acétamipride et de lambda cyhalothrine, chez le poisson Oreochromis niloticus exposé à des doses chroSniques. Mémoire de master, Ecole de Biologie, Université Catholique de Louvain, Belgique, p. 70.
Sarr SM, Thiam A, Faye El-H, Sene M, Ndiaye M. 2015. Production d'alevins de Tilapia (Oreochromis niloticus) avec trois aliments à base de sous-produits agro-industriels au Nord du Sénégal. International Journal of Biological and Chemical Sciences, 9(5): 25982606.DOI: http://dx.doi.org/10.4314/ijbcs.v9i5.29

Soclo HH, Azontonde AH, Dovonou LF, Djibril R, Sagbo AU. 2004. Etude de l'impact de l'utilisation des engrais chimiques et des pesticides par les populations riveraines sur les écosystèmes (eaux de surfaces, substrats des réserves de faune) dans les complexes des aires protégées de la Pendjari et du W. CENAGREF, Cotonou, p.135.

Soderlund DM, Clark JM, Sheets LP, Mullin LS, Piccirillo VJ, Sargent D, Stevens JT, Weiner ML. 2002. Mechanisms of pyrethroid neurotoxicity: implications for cumulative risk assessment. Toxicology, 171: 3-59.

Yèhouénou A, Pazou E. 2005. Les résidus de pesticides chimiques de synthèse dans les eaux, les sédiments et les espèces aquatiques du bassin versant du fleuve Ouémé et du lac Nokoué, Thèse de doctorat, Ecole Polytechnique d'Abomey-Calavi, Bénin, p. 214.

Zoumenou B, Aïna MP, Agbohessi P, Imorou Toko I, Scippo M-L. 2015. Effets toxicologiques et méthodes d'analyse de la lambda-cyhalothrine et de l'acétamipride utilisés dans la protection phytosanitaire du cotonnier au Bénin. International Journal of Biological and Chemical Sciences, 9(4): 2184-2199. DOI:

http://dx.doi.org/10.4314/ijbcs.v9i4.38. 